\title{
Control strategies for an octopus-like soft manipulator
}

\author{
Simone Cacace ${ }^{1}$, Anna Chiara Lai ${ }^{2}{ }^{a}$ a and Paola Loreti ${ }^{2}$ \\ ${ }^{1}$ Dipartimento di Matematica e Fisica, Università degli Studi Roma Tre, Largo S. Murialdo, 1, 00154 Roma, Italy \\ ${ }^{2}$ Dipartimento di Scienze di Base e Applicate per l'Ingegneria, Sapienza Università di Roma, Via A. Scarpa, 16, 00161 \\ Roma, Italy \\ cacace@mat.uniroma3.it,\{anna.lai,paola.loreti\}@sbai.uniroma1.it
}

\begin{abstract}
Keywords: Soft manipulators, octopus arm, control strategies.
Abstract: We investigate a reachability control problem for a soft manipulator inspired to an octopus arm. Cases modelling mechanical breakdowns of the actuators are treated in detail: we explicitly characterize the equilibria, and we provide numerical simulations of optimal control strategies.
\end{abstract}

This is a pre-copy-editing, author-produced PDF of an article accepted for publication in following peer review. The definitive publisher-authenticated version 16th International Conference on Informatics in Control, Automation and Robotics (1) 82-90 (2019) is available online at: www.scitepress.org.

\section{INTRODUCTION}

In this paper we investigate a control theoretical framework for a soft manipulator model, and we present related numerical simulations. Our model allows to deal with the case in which only a portion of the manipulator is controlled, while the remaining part of the device is driven by internal reaction forces only. This scenario includes mechanical breakdowns, or a voluntary deactivation for energy saving purposes: its investigation is one of the main novelties also with respect to (?), where the model is originally introduced.

Soft manipulators attracted the interest of researchers due to their ability to adapt to constrained enviroments, and their potential suitability for humanrobot interactions (?; ?). Starting from the first works on hyper-redundant manipulators (?; ?), the challenging motion planning of tentacle-like soft manipulators was attacked by several points of view: among many others, we refer to (?) for the study of the kinematics, to (?; ?) for a discrete dynamical model, to (?; ?; ?) for a number theoretic approach, and to (?) for a machine learning implementation. To the best of our

a (D) https://orcid.org/0000-0003-2096-6753 knowledge, the approach in the present paper, based on optimal control theory of PDEs (see for instance (?)), appears to the be new.

Our device is modelled as an inextensible elastic string with curvature constraints (i.e. the device cannot bend over a fixed threshold), and whose curvature is pointwise forced by a control term, modelling an angular elastic internal force. The resulting dynamics is an evolutive controlled non-linear fourth order partial differential equation, generalizing the classical Euler-Bernoulli equation. At this stage of our investigation, we adopt some simplifications in the model -we are dealing with a theoretical object, a fully responsive, noise free planar manipulator with infinite degrees of freedom- but this allows us to go into the deep in the optimization process, by providing optimal open loop controls for a reachability problem.

The paper is organized as follows. In Section ?? we introduce our model in full generality and we discuss some particular parameter settings. In Section ?? we investigate the problem of steering the tip of the manipulator to a target point, while minimizing a quadratic cost on the controls and the kinetic energy at final time of the manipulator. Section ?? contains some numerical simulations and finally in Section ?? we draw our conclusions.

\section{A CONTROL MODEL FOR A SOFT MANIPULATOR}

Our goal is to describe the dynamics of a soft manipulator encompassing the following features: 
Table 1: Exact constraint equations and related elastic potentials derived from penalty method. The functions $v$ and $\mu$ represent non-uniform elastic constants.

\begin{tabular}{|l|l|l|}
\hline Constraint & $\begin{array}{l}\text { Constraint } \\
\text { equation }\end{array}$ & $\begin{array}{l}\text { Penalization } \\
\text { elastic potential }\end{array}$ \\
\hline Inextensibility & $\left|q_{s}\right|=1$ & None \\
Curvature & $\left|q_{s s}\right| \leq \omega$ & $\mathrm{v}\left(\left|q_{s s}\right|^{2}-\omega^{2}\right)_{+}^{2}$ \\
Control & $q_{s} \times q_{s s}=\omega u$ & $\mu\left(\omega u-q_{s} \times q_{s s}\right)^{2}$ \\
\hline
\end{tabular}

- inextensibility: the manipulator can bend but not stretch longitudinally.

- bending moment: the soft structure of the device resists to bending via an angular elastic potential.

- bending constraint: the manipulator cannot bend over a fixed threshold

- bending control: a time-varying, internal angular elastic force is applied in order to pointwise force the bending of the device.

From a morphological point of view, we regard the manipulator as a three-dimensional body with an axial symmetry, a non-uniform thickness and a fixed endpoint. In what follows we build up a system of PDEs modelling the evolution of the curve on the plane representing the symmetry axis. From a physical point of view, such an axis is modelled as an inextensible string, whose mass represents the mass of the whole manipulator. Also bending constraints (and controls) of the manipulator are projected on the axis: they are identified by suitably weighted curvature constraints. For instance, the bending constraint is translated into forcing the curvature of the axis under a fixed (nonuniform) threshold $\omega$; the bending control is translated into forcing the signed curvature to the quantity $\omega u$, where $u \in[-1,1]$ is the control map. The relation between the original three-dimensional problem and the projected one is further discussed in Section ?? below. In what follows, we focus on the symmetry axis evolution. The unknowns of our problem are the curve $q(s, t):[0,1] \times \mathbb{R}^{+} \rightarrow \mathbb{R}^{2}$ parametrizing the symmetry axis of the manipulator in arclength coordinates, and the associated inextensibility multiplier $\sigma(s, t) \in \mathbb{R}$. We denote by $q_{s}, q_{s s}, q_{t t}$ partial derivatives in space and time respectively. The quantity $\left|q_{s s}\right|$ represents the curvature of $q$, whereas the product $q_{s} \times q_{s s}:=q_{s} \cdot q_{s s}^{\perp}$ represents the signed curvature, where the symbol $q_{s s}^{\perp}$ denotes the counter-clockwise orthogonal vector to $q_{s s}$. With these notations, we summarize the constraints described above in Table ??. Note that also the bending moment allows for a description in terms of curvature constraints: imposing the constraint $\left|q_{s s}\right|=0$ by penalty method yields the elastic potential $\varepsilon\left|q_{s s}\right|^{2}$, for some elastic (constant in time) function $\varepsilon(s)$. We then consider the Lagrangian

$$
\begin{aligned}
\mathcal{L}(q, \sigma):=\int_{0}^{1} & \left(\frac{1}{2} \rho\left|q_{t}\right|^{2}-\frac{1}{2} \sigma\left(\left|q_{s}\right|^{2}-1\right)\right. \\
& -\frac{1}{4} v\left(\left|q_{s s}\right|^{2}-\omega^{2}\right)_{+}^{2}-\frac{1}{2} \varepsilon\left|q_{s s}\right|^{2} \\
& \left.-\frac{1}{2} \mu\left(\omega u-q_{s} \times q_{s s}\right)^{2}\right) d s
\end{aligned}
$$

whose integral terms respectively represent the kinetic energy ( $\rho$ is the mass density), the exact inextensibility constraint ( $\sigma$ is the Lagrange multiplier), the curvature constraint, the bending moment, and the curvature control. Equation of motions are then derived via the least action principle - see (?). They result in the following system:

$$
\left\{\begin{array}{l}
\rho q_{t t}=\left(\sigma q_{s}-H q_{s s}^{\perp}\right)_{s}-\left(G q_{s s}+H q_{s}^{\perp}\right)_{s s} \\
\left|q_{s}\right|^{2}=1
\end{array}\right.
$$

for $(s, t) \in(0,1) \times(0, T)$. The map $G:=$ $G[q, v, \varepsilon, \omega]=\varepsilon+v\left(\left|q_{s s}\right|^{2}-\omega^{2}\right)+$ encompasses the bending moment and the curvature constraint $(\cdot)_{+}$denotes the positive part. The map $H:=$ $H[q, \mu, u, \omega]=\mu\left(\omega u-q_{s} \times q_{s s}\right)$ represents the control term. Equation (??) is completed with suitable initial data, and with the following boundary conditions for $t \in(0, T)$ :

- fixed endpoint conditions:

$$
\left\{\begin{array}{l}
q(0, t)=(0,0) \\
q_{s}(0, t)=-(0,1)
\end{array}\right.
$$

- free endpoint conditions:

$$
\begin{cases}q_{s s}(1, t)=0 & \text { (zero bending moment) } \\ q_{s s s}(1, t)=0 & \text { (zero shear stress) } \\ \sigma(1, t)=0 & \text { (zero tension boundary). }\end{cases}
$$

Note that the fixed endpoint conditions are a modelling choice, while the free endpoint conditions follow from the stationarity conditions for the Lagrangian $\mathcal{L}$.

For technical details on the derivation of the equations we refer to (?).

\subsection{Model parameter settings}

We propose a parameter tuning in order to encompass, in the one-dimensional model, the morphology of the original three-dimensional manipulator. The key morphological assumptions on the manipulator are the following: axial symmetry and uniform mass density $\rho_{v}$. In particular, we assume that in its position at rest 
(i.e. when its symmetry axis has uniformly zero curvature) the manipulator is a solid of rotation, generated by the function $d(s), s \in[0,1]$; we call $\Omega(s)$ the circle of radius $d(s)$ representing the cross section of the manipulator at the point $s \in[0,1]$ of its axis. Note that $d$ measures the thickness of the manipulator: in octopus arm shaped manipulators, $d$ is a decreasing function. Choosing $\rho(s):=\pi \rho_{v} d(s)^{2}$ corresponds to concentrate the mass of $\Omega(s)$ on its barycentre.

In our model, bending the manipulator determines a compression/dilatation of the soft, elastic material composing its body, for which we assume an uniform yield point, i.e., the pointwise elastic forces must be uniformly bounded by some $F_{\max }$ in order to avoid inelastic deformations. Now, due to the axial symmetry, for every $s \in[0,1]$, the maximal angular elastic force in $\Omega(s)$ is attained on its boundary and it reads $F(s)=e\left|q_{s s}\right| d(s)$, where $e$ is the elastic constant of the material. Therefore the constraint $F(s) \leq F_{\max }$ is equivalent to the curvature constraint $\left|q_{s s}\right| \leq \omega$, where we set $\omega(s)=F_{\text {max }} /(e d(s))$.

\subsubsection{On mass distributions with exponential decay}

In our numerical simulations, we assume $\rho$ to have an exponential decay. By the arguments above, this choice is consistent with the case of three dimensional structures whose thickness decays exponentially, and it can be viewed as an interpolation of self-similar, discrete structures (?). In turn, self-similarity assumption has several advantages from both a practical and theoretical point of view. Indeed, asking for a manipulator to be self-similar simply means to be composed by identical, rescaled modules. From a geometrical point of view, self-similarity is a powerful assumption allowing, via fractal geometry techniques (?), for a detailed investigation of inverse kinematics of the manipulator, see for instance (?).

\subsection{Control parameter settings}

We now show how to set the control parameters to model a mechanical breakdown of the actuators.

We recall that the controlled elastic potential is

$$
U[q, \mu, u, \omega]:=\frac{1}{2} \mu\left(\omega u-q_{s} \times q_{s s}\right)^{2} .
$$

and the associated elastic force is

$$
F[q, \mu, u, \omega]:=-\left(H q_{s s}^{\perp}\right)_{s}-\left(H q_{s}^{\perp}\right)_{s s}
$$

where $H=\mu\left(\omega u-q_{s} \times q_{s s}\right)$. Clearly, to choose $\mu \equiv 0$ is equivalent to neglect $F$, and it yields the uncontrolled dynamics:

$$
\rho q_{t t}=\left(\sigma q_{s}\right)_{s}-\left(G q_{s s}\right)_{s s}
$$

More generally, to set $\mu(s)=0$ for $s \in\left[s_{1}, s_{2}\right]$ means that the portion of $q$ between $\left[s_{1}, s_{2}\right]$ is uncontrolled: it evolves according to (??) with (time dependent, controlled) boundary conditions in $q\left(s_{1}, t\right)$ and $q\left(s_{2}, t\right)$.

We conclude with some remarks on how the control deactivation affects the equilibria of the system. A general formula for the equilibria of (??) is provided in (?): if $\mu(1)=\mu_{s}(1)=0$, then the signed curvature $\kappa:=q_{s} \times q_{s s}$ of the (unique) equilibrium $q$ of (??) is proportional to $\mu$, precisely it satisfies

$$
\kappa(s)=\mu(s) \frac{\omega(s) u(s)}{\mu(s)+\varepsilon(s)} \quad s \in(0,1) .
$$

In particular for $\mu \equiv 0$ the equilibrium is given by $q(s, t) \equiv(0,-s)$ for all $(s, t) \in[0,1] \times(0, T)$. More generally, if $q$ is uncontrolled in $\left[s_{1}, s_{2}\right]$, i.e., $\mu(s)=0$ for all $s \in\left[s_{1}, s_{2}\right]$, then $\left|q_{s s}\right|=|\kappa(s)|=0$, that is the corresponding portion of the device at the equilibrium is arranged in a straight line.

\section{THE REACHABILITY OPTIMAL CONTROL PROBLEM}

In this section we address the problem of steering the tip of the device -i.e., $q(1, t)-$ to a target point $q^{*} \in \mathbb{R}^{2}$, using controls that optimize accuracy, steadiness and energy consumption. In the framework of optimal control theory, this problem can be restated as a constrained minimization of a cost functional involving:

- the tip-target distance: we want the tip to reach and remain close to the target;

- a quadratic cost on the controls, that is we want to force the device - precisely, its symmetry axis- to bend as least as possible.

- the kinetic energy of the whole manipulator at final time.

More formally, given $q^{*} \in \mathbb{R}^{2}$ and $T, \tau>0$, we want to minimize the functional

$$
\begin{aligned}
\mathcal{J}(q, u) & =\frac{1}{2 \tau} \int_{0}^{T}\left|q(1, t)-q^{*}\right|^{2} d t \\
& +\frac{1}{2} \int_{0}^{T} \int_{0}^{1} u^{2} d s d t \\
& +\frac{1}{2} \int_{0}^{1} \rho(s)\left|q_{t}(s, T)\right|^{2} d s,
\end{aligned}
$$

among all the controls $u:[0,1] \times[0, T] \rightarrow[-1,1]$, and subject to the symmetry axis dynamics (??). The first two terms in $\mathcal{J}$ account respectively for the tip-target distance and the quadratic cost on the controls, during 
all the evolution, whereas the last term corresponds to the kinetic energy at the final time $T$.

In what follows we give the first order optimality conditions for this control problem, that is we write a system of PDEs, called the adjoint system, whose unknowns are the stationary points $(q, \sigma, u)$ of $\mathcal{I}$ (subject to the symmetry axis dynamics), and the related multipliers $(\bar{q}, \bar{\sigma})$ called the adjoint states. Roughly speaking, if a control $u^{*}$ is optimal, then the adjoint system admits a solution of the form $\left(q, \sigma, u^{*}, \bar{q}, \bar{\sigma}\right)$. The adjoint system is composed by a variational inequality for the optimal control and four PDEs: two of them describe the evolution of the adjoint states, and two of them are simply the dynamics the system is subject to, i.e., the equations of motion of the symmetry axis (??). Following (?), the adjoint states equations are:

$$
\left\{\begin{array}{c}
\rho \bar{q}_{t t}=\left(\sigma \bar{q}_{s}-H \bar{q}_{s s}^{\perp}\right)_{s}-\left(G \bar{q}_{s s}+H \bar{q}_{s}^{\perp}\right)_{s s} \\
\quad+\left(\bar{\sigma} q_{s}-\bar{H} q_{s s}^{\perp}\right)_{s}-\left(\bar{G} q_{s s}+\bar{H} q_{s}^{\perp}\right)_{s s} \\
\bar{q}_{s} \cdot q_{s}=0
\end{array}\right.
$$

for $(s, t) \in(0,1) \times(0, T)$, where

- the maps $G$ and $H$ are defined in Section ??

- the maps $\bar{G}$ and $\bar{H}$ are the linearisations of $G$ and $H$, respectively. In particular

$$
\begin{gathered}
\bar{G}[q, \bar{q}, \nu, \omega]=g[q, v, \omega] q_{s s} \cdot \bar{q}_{s s}, \\
\bar{H}[q, \bar{q}, \mu]=\mu\left(\bar{q}_{s} \times q_{s s}+q_{s} \times \bar{q}_{s s}\right),
\end{gathered}
$$

where $g[q, v, \omega]=2 v \mathbf{1}\left(\left|q_{s s}\right|^{2}-\omega^{2}\right)$ and $\mathbf{1}(\cdot)$ stands for the Heaviside function, i.e. $\mathbf{1}(x)=1$ for $x \geq 0$ and $\mathbf{1}(x)=0$ otherwise.

System (??) is completed with the following final and boundary conditions, derived as well from the optimality conditions:

- final conditions:

$$
\bar{q}(s, T)=-q_{t}(s, T), \quad \bar{q}_{t}(s, T)=0
$$

for $s \in(0,1)$. We point out that initial conditions for $q$ are replaced in the adjoint system with final conditions for $\bar{q}$ : this is a quite fair consequence of the fact that the optimization process takes into account the dynamics on the whole timeline $(0, T)$.

- fixed endpoint boundary conditions:

$$
\bar{q}(0, t)=0, \quad \bar{q}_{s}(0, t)=0
$$

for $t \in(0, T)$ - remark the symmetry with the fixed endpoint conditions on $q$;

- free endpoint boundary conditions:

$$
\left\{\begin{array}{l}
\bar{q}_{s s}(1, t)=0 \\
\bar{q}_{s s s}(1, t)=\frac{1}{\tau \varepsilon}\left(\left(q-q^{*}\right) \cdot q_{s}^{\perp}\right) q_{s}^{\perp}(1, t) \\
\bar{\sigma}(1, t)=-\frac{1}{\tau}\left(q-q^{*}\right) \cdot q_{s}(1, t)
\end{array}\right.
$$

for $t \in(0, T)$. Note that we recover the zero bending moment condition, as for the free endpoint of $q$. On the other hand, as one may expect, both the shear stress condition on $\bar{q}$ and the adjoint tension boundary condition on $\bar{\sigma}$ are affected by the fact that the system is forced towards the target $q^{*}$.

Finally, the variational inequality for the control is

$$
\int_{0}^{T} \int_{0}^{1}(u+\omega \bar{H}[q, \bar{q}])(v-u) d s d t \geq 0 .
$$

for every $v:[0,1] \times[0, T] \rightarrow[-1,1]$, which provides, in a weak sense, the variation of the functional $\mathcal{I}$ with respect to $u$, subject to the constraint $|u| \leq 1$.

\section{NUMERICAL SIMULATIONS}

The optimal control problem discussed in Section ?? is approached in two phases. At a first stage we look at a stationary optimal control problem, involving the equilibria of the system (??). Then such stationary solutions are used as initial guess for the original dynamic optimal control problem, i.e., for the numerical solution of the system composed by (??), (??) and (??).

\subsection{Stationary optimal control problem}

First of all we remark that if $(q, \sigma)$ is an equilibrium for the symmetry axis equation (??) and if $\mu(1)=$ $\mu_{s}(1)=0$, then $q$ is the solution of the following second order ODE:

$$
\begin{cases}q_{s s}=\frac{\mu \omega}{\mu+\varepsilon} u q_{s}^{\perp} & \text { in }(0,1) \\ \left|q_{s}\right|^{2}=1 & \text { in }(0,1) \\ q(0)=(0,0) & \\ q_{s}(0)=-(0,1) . & \end{cases}
$$

The stationary optimal control problem is the following

$$
\min \left\{\frac{1}{2} \int_{0}^{1} u^{2} d s+\frac{1}{2 \tau}\left|q(1)-q^{*}\right|^{2}\right\},
$$

subject to (??) and to $|u| \leq 1$. Note that the above cost functional is the stationary version of $\mathcal{H}$ : a quadratic cost on the controls and a tip-target distance are still involved, whereas time integrals and time-dependent terms as the kinetic energy are clearly neglected. Using the first two equations of (??), we have $\left|q_{s s}\right|=$ $\bar{\omega}|u|$, where $\bar{\omega}:=\mu \omega /(\mu+\varepsilon)$. Therefore we can get rid of the dependence from $u$ in (??) and look for the 


\begin{tabular}{|l|l|}
\hline Parameter description & Setting \\
\hline mass distribution & $\rho(s)=\exp (-s)$ \\
bending moment & $\varepsilon(s)=10^{-3}(1-0.9 s)$ \\
curvature constraint & $\mathrm{v}(s)=10^{-3}(1-0.09 s)$ \\
penalty & $\mu(s)=(1-s) e^{-0.1 \frac{s^{2}}{1-s^{2}}}$ \\
$\begin{array}{l}\text { curvature control } \\
\text { penalty } \\
\text { curvature constraint }\end{array}$ & $\omega(s)=3 \pi\left(1+s^{2}\right)$ \\
\hline $\begin{array}{l}\text { target point } \\
\text { target penalty }\end{array}$ & $q^{*}=(0.3563,-0.4423)$ \\
\hline Discretization step & $\Delta_{s}=0.05$ \\
\hline
\end{tabular}

Table 2: Global parameter settings.

solutions of the following Euler elastica's like problem:

$$
\begin{aligned}
& \min \left\{\frac{1}{2} \int_{(0,1) \backslash I} \frac{1}{\overline{\bar{\omega}}^{2}}\left|q_{s s}\right|^{2} d s+\frac{1}{2 \tau}\left|q(1)-q^{*}\right|^{2}\right\} \\
& \text { subject to } \begin{cases}\left|q_{s}\right|^{2}=1 & \text { in }(0,1) \\
\left|q_{s s}\right| \leq \bar{\omega} & \text { in }(0,1) \\
q(0)=(0,0) & \\
q_{s}(0)=-(0,-1),\end{cases}
\end{aligned}
$$

where we set $I:=\{s \in(0,1) \mid \mu(s)=0\}$. In other words we are looking for the curve $q$-with length 1 and curvature bounded by $\bar{\omega}-$ minimizing the total curvature and the distance of its tip from $q^{*}$. Dotmultiplying by $q_{s}^{\perp}$ both sides of the first equation of (??), we obtain that the optimal control for the original stationary problem (??) is then given by

$$
u(s)= \begin{cases}\frac{q_{s s}(s) \cdot q_{s}^{\perp}(s)}{\overline{\bar{\omega}}(s)} & \text { if } \mu(s) \neq 0, \\ 0 & \text { otherwise. }\end{cases}
$$

Note that in (?) we assumed $\mu>0$ in $(0,1)$. Here we also consider the case $\mu(s)=0$. As a matter of fact, for $\mu(s)=0$, the dynamics and its equilibria are independent from $u(s)$ : it can be arbitrarily chosen. The particular choice $u(s)=0$ in $I$ allows the cost functional (??) to be consistent also with the case of uncontrolled regions.

To numerically solve (??) we encompass the constraints $\left|q_{s}\right|=1$ and $\left|q_{s s}\right| \leq \bar{\omega}$ via an augmented Lagrangian method, and we discretize it with a finite difference scheme. The non-linear terms of the resulting discrete system of equations are treated via a quasi Newton's method. Parameters settings are reported in Table ??.

We investigate the problem in three scenarios, see Table ??: in the first we assume that $\mu$ is positive in $(0,1)$, in the second and third experiment we neglect $\mu$ in some sub-intervals of $(0,1)$. The latter cases represent situations in which we cannot control a portion of the device - see Section ??. In particular, for a

\begin{tabular}{|l|l|}
\hline Test & Control deactivation region \\
\hline Test 1 & $I=\emptyset$ \\
Test 2 & $I=[0.35,0.65]$ \\
Test 3 & $I=[0.25,0.4] \cup[0.6,0.75]$ \\
\hline
\end{tabular}

Table 3: Control deactivation settings related to $\mu_{I}$.

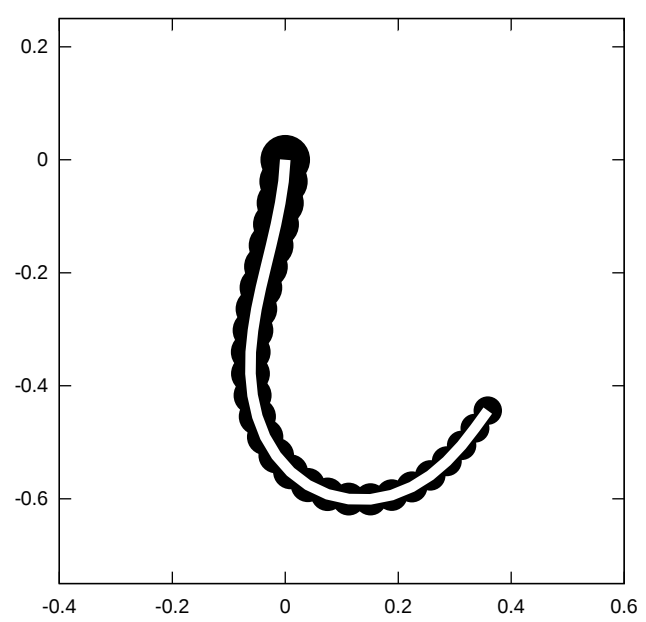

(a)

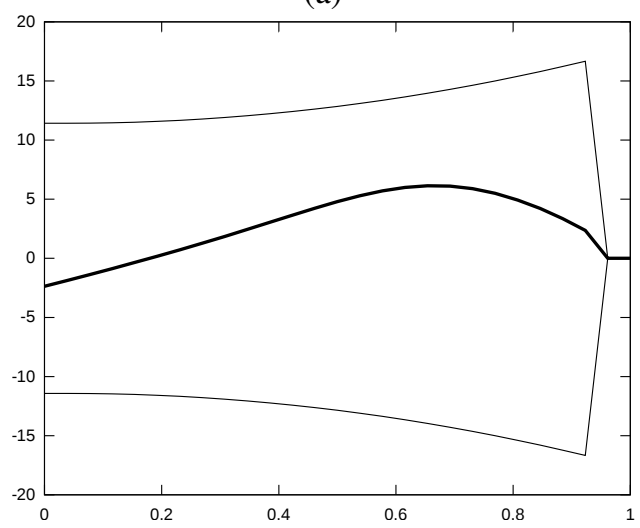

(b)

Figure 1: In (a) the solution $q$ of Test 1 , in (b) the related signed curvature $\kappa(s)$ (bold line) and curvature constraints $\pm \bar{\omega}$ (thin lines).

given $I \subset(0,1)$, we employ the following curvature control penalty parameter

$$
\mu_{I}(s)= \begin{cases}0 & \text { if } s \in I \\ \mu(s) & \text { otherwise }\end{cases}
$$

In Figure ?? we report the results of Test 1 -see Table ??. In Figure ?? and ?? we show the results of Test 2 and Test 3, respectively. In these cases the controls are in-actuated in a subset of $(0,1)$ : the corresponding regions are depicted in red. The particular choice of $q^{*}$ allows for it to be reached in all these three cases, but clearly, with different optimal solutions. 


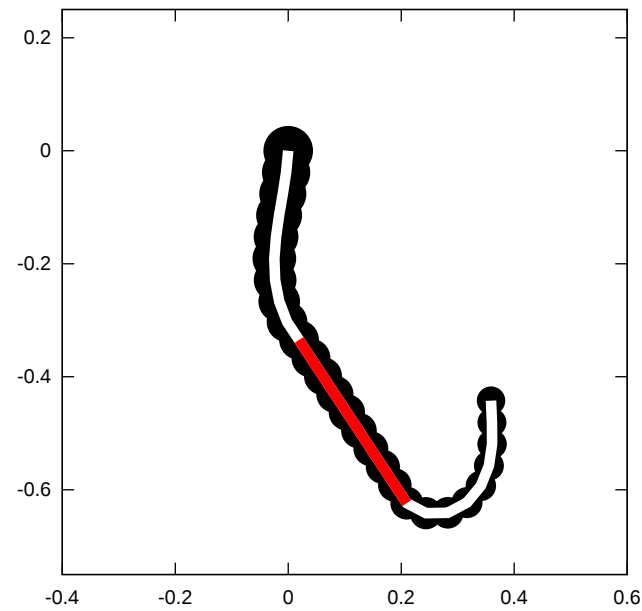

(a)

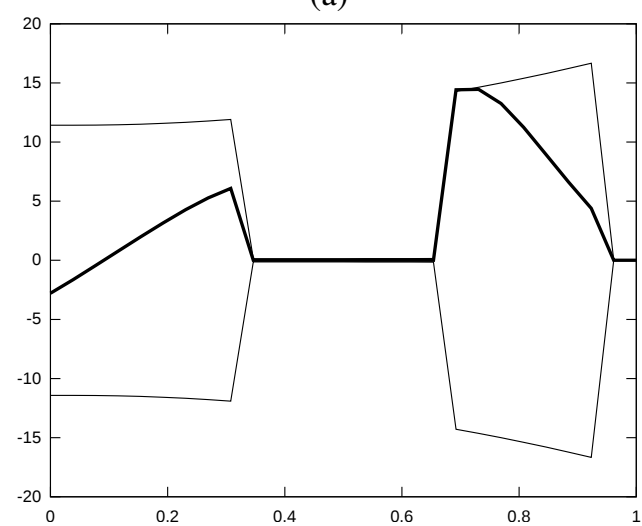

(b)

Figure 2: In (a) the solution $q$ of Test 2, in (b) the related signed curvature $\kappa(s)$ (bold line) and curvature constraints $\pm \bar{\omega}$ (thin lines).

\subsection{Dynamic optimal control problem}

We address the minimization of the cost functional $\mathcal{g}$ subject to a dissipative version of the dynamical system (??):

$$
\begin{aligned}
\rho q_{t t}= & \left(\sigma q_{s}-H q_{s s}^{\perp}\right)_{s}-\left(G q_{s s}+H q_{s}^{\perp}\right)_{s s} \\
& -\beta q_{t}-\gamma q_{s s s s t} .
\end{aligned}
$$

In particular, the term $-\beta q_{t}$ represents an environmental viscous friction proportional to the velocity; the term $-\gamma q_{\text {sssst }}$ an internal viscous friction, proportional to the change in time of the curvature. This implies that if we plug in (??) the optimal stationary (constant in time) control $u$ given in Equation (??), then the system converges as $T \rightarrow+\infty$ to the optimal stationary equilibrium $q$ described in Section ?? - see Equation (??). Here we look for time-varying optimal controls, i.e., for solutions $u^{*}$ of the dynamic optimal control problem described in Section ??. The adjoint

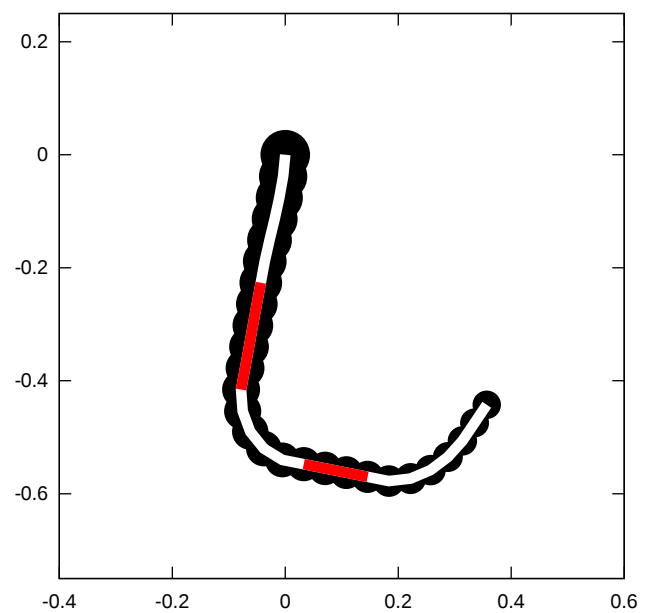

(a)

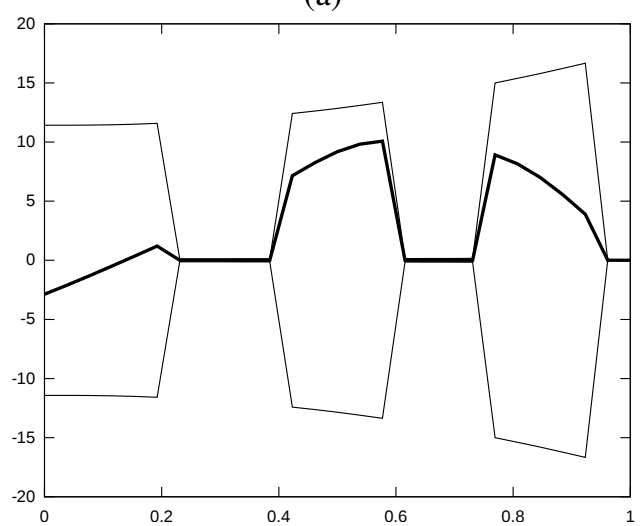

(b)

Figure 3: In (a) the solution $q$ of Test 3, in (b) the related signed curvature $\kappa(s)$ (bold line) and curvature constraints $\pm \bar{\omega}$ (thin lines).

\begin{tabular}{|l|l|}
\hline Parameter description & Setting \\
\hline Environmental friction & $\beta(s)=\beta(s):=2-s$ \\
Internal friction & $\gamma(s):=10^{-6}(2-s)$ \\
Final time & $T=2$ \\
Time discretization step & $\Delta_{t}=0.001$ \\
\hline
\end{tabular}

Table 4: Dynamic parameter settings.

states equations in the dissipative version read:

$$
\left\{\begin{array}{c}
\rho \bar{q}_{t t}=\left(\sigma \bar{q}_{s}-H \bar{q}_{s s}^{\perp}\right)_{s}-\left(G \bar{q}_{s s}+H \bar{q}_{s}^{\perp}\right)_{s s} \\
\quad+\left(\bar{\sigma} q_{s}-\bar{H} q_{s s}^{\perp}\right)_{s}-\left(\bar{G} q_{s s}+\bar{H} q_{s}^{\perp}\right)_{s s} \\
\quad+\beta \bar{q}_{t}+\gamma \bar{q} \bar{q}_{s s s t} \\
\bar{q}_{s} \cdot q_{s}=0
\end{array}\right.
$$

for $(s, t) \in(0,1) \times(0, T)$.

The adjoint system composed by (??), (??) and (??) can be discretized using a standard finite difference scheme in space-time, then solved by an adjointbased gradient descent method. The key idea is the 


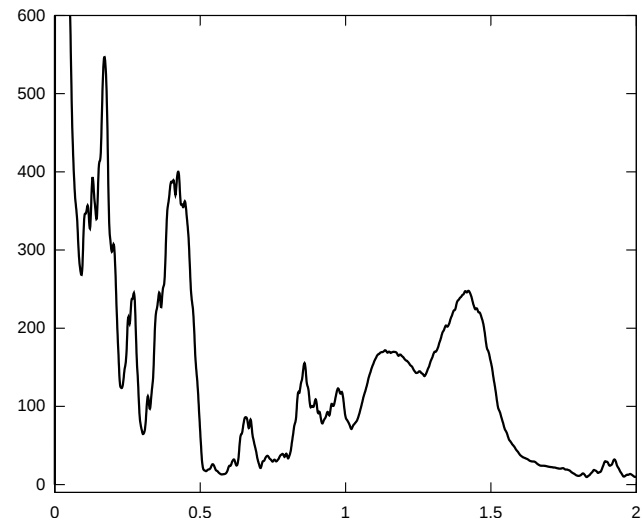

(a)

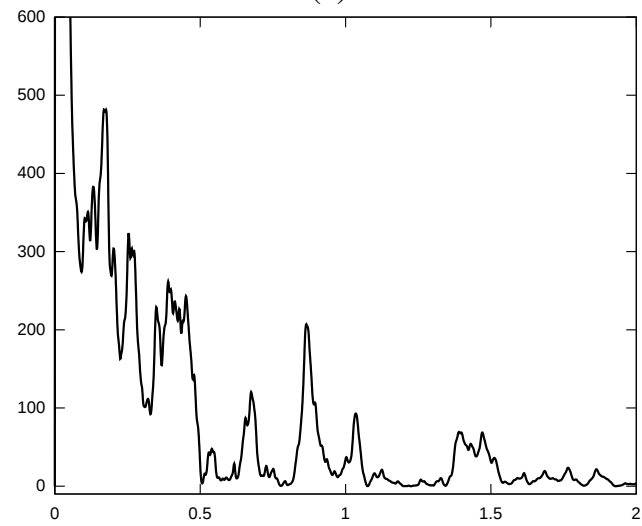

(b)

Figure 4: Time evolution of target energy $g_{q^{*}}$ for the static (a) and dynamic (b) optimal control.

following: starting from an initial guess $u$ given by the stationary optimal control, we first solve the equation of motion (??) forward in time. Then the solutioncontrol triplet $(q, \sigma, u)$ is plugged into (??) which is solved backward in time. Finally, we use the vector $(q, \sigma, u, \bar{q}, \bar{\sigma})$ in (??) to update the value of the $u$. The procedure is iterated up to convergence on the control.

We assume that $\mu(s)=0$ in a subinterval $I=$ $[0.35,0.65]$ of $(0,1)-$ this choice corresponding to Test 2 in Section ??, see Table ?? and Figure ??. In other words, we are modelling the scenario in which approximately one third of the central actuators of the device are out of order. The parameters related to the dynamical aspects, in particular those involved by friction forces and time discretization are reported in Table ??. The other parameters are set as in Section ??, see Table ??. We compare the performances of optimal stationary and dynamic controls in terms of the evolution of the three components of our integral cost: the tip-target distance, the control energy and the kinetic energy.
In Figure ?? we plot the function

$$
\mathcal{I}_{q^{*}}(t):=\left|q(1, t)-q^{*}\right|^{2}
$$

representing the tip-target distance at time $t \in[0,2]$, in both cases of statically and dynamically optimized controls. More precisely, in Figure ??(a) we plugged in the system (??) a constant in time control $u^{s}$, that we call static optimal control, given by the solution of the stationary control problem (??). We then considered the resulting trajectory $q(s, t)$ and measured the tip-target distance $g_{q^{*}}(t)$. In Figure ??(b) we see the tip-target distance of the trajectory $q$ obtained by numerically solving the adjoint system, that is the trajectory corresponding to a time-dependent optimal control $u^{d}$ that we call in the sequel dynamic optimal control. In agreement with the theoretical setting, in particular with the strong dependence of the cost functional $\mathcal{I}$ on the tip-target distance (see also the value for the weight $\tau$ in Table ??), the dynamical optimization process yields, up to some oscillations, a much lower $\mathcal{J}_{q^{*}}(t)$ than the stationary case. We also remark the damping of the oscillations of $g_{q^{*}}(t)$ in the dynamical case: this confirms that the dynamical optimization process tends to reach and stay close to the target as soon as possible.

In Figure ?? we plot the function

$$
g_{u}(t):=\int_{0}^{1} u^{2}(t) d s
$$

representing the quadratic cost on the controls at time $t \in[0,2]$. In the case of static controls, $\mathcal{J}_{u^{s}}(t)$ is constant by construction. The dynamic optimal controls $\mathcal{g}_{u^{d}}(t)$ are on average smaller than $\mathcal{J}_{u^{s}}(t)$ : this implies that the dynamical optimization process actually reduces the total cost of the controls

$$
\int_{0}^{T} \mathcal{J}_{u}(t) d t
$$

with respect to the stationary optimization. Also note that the dynamic optimal control $u^{d}$ yields a large variation of its cost $\mathcal{g}_{u^{d}}(t)$ at the beginning of the evolution, while stabilizing it around the static control cost $\mathcal{J}_{u^{s}}(t)$ in a second time. This implies that the optimization process tends to concentrate the variation of controls at the beginning, while the second part of the evolution is demanded to small adjustments, confirming what we already remarked by looking at the tip-target distance evolution.

Finally, in Figure ?? we plot the function

$$
\mathcal{J}_{v}(t):=\frac{1}{2} \int_{0}^{1} \rho(s)\left|q_{t}(s, t)\right|^{2} d s
$$

representing the kinetic energy of the whole axis at time $t \in[0,2]$ in the cases of stationary and dynamic optimal controls. Note that the evolutions of the $\mathcal{I}_{v}$ 


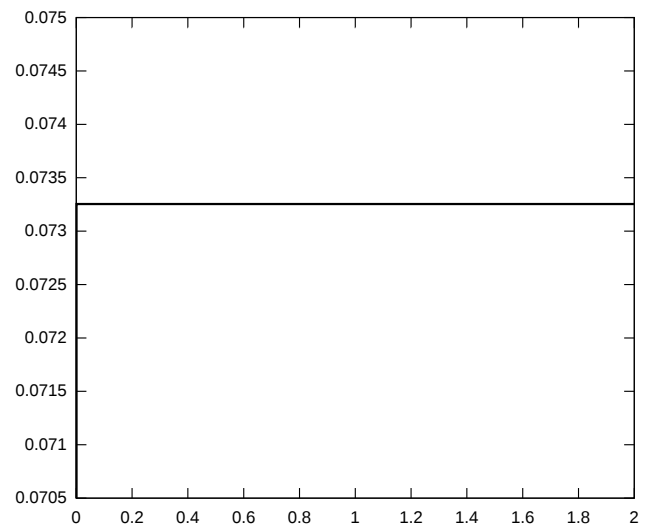

(a)

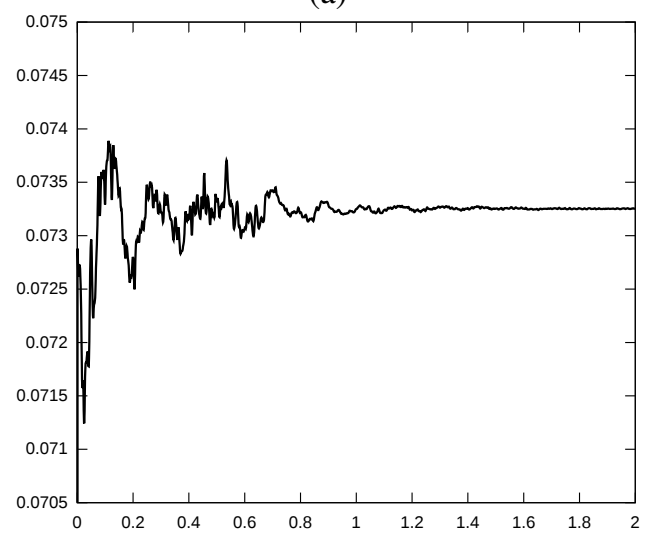

(b)

Figure 5: Time evolution of control energy $\mathcal{J}_{u}$ for the stationary (a) and dynamic (b) optimal control.

are comparable, but at final time $T$, dynamical optimal controls yield a kinetic energy $\mathcal{I}_{v}(T)$ lower than the one associated to the stationary optimal controls. This is consistent with the fact that the cost functional $\mathcal{I}$ depends only on the kinetic energy at final time. Finally note that, since the system is converging to an equilibrium due to frictional forces, $\mathcal{I}_{v}(t) \rightarrow 0$ as $t \rightarrow+\infty$ : it is then reasonable to expect a larger time frame to improve the performances of the kinetic energy in both dynamic and stationary cases. For the dynamically controlled case only, steadiness can also be traded with accuracy by increasing the weight of the term $\mathcal{I}_{v}(T)$ in the functional $\mathcal{I}$.

\section{CONCLUSIONS}

In this paper we addressed the optimal control of a planar soft manipulator, modelled as an inextensible elastic string with non-uniform mass, curvature constraints and curvature controls. Parameters are set in order to encompass in the one-dimensional model the

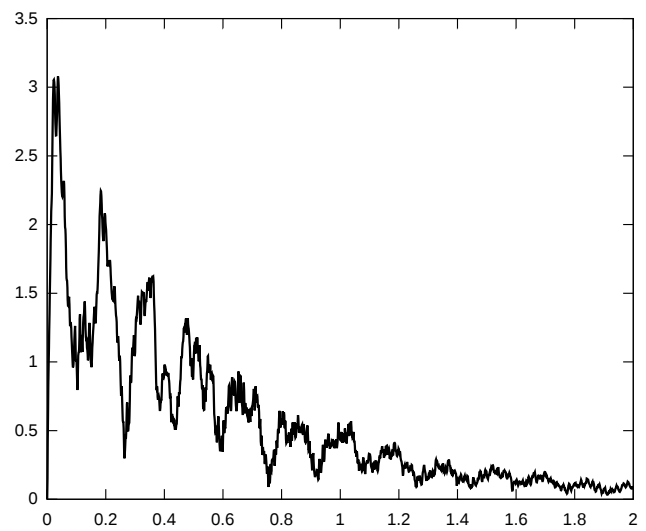

(a)

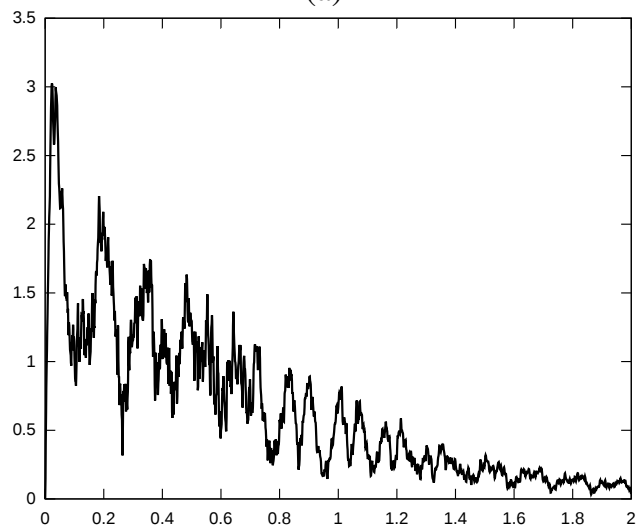

(b)

Figure 6: Time evolution of kinetic energy $\mathcal{I}_{v}$ for the static (a) and dynamic (b) optimal control.

morphology of a three-dimensional manipulator. We looked in particular to the case in which a part of the device is uncontrolled and, consequently, driven only by internal reaction forces. Optimal open loop controls are obtained via a constrained minimization of a cost functional in both the stationary and dynamic case. Numerical simulations are provided in the special case of partially actuated controls. They agree with the theoretical control model.

A challenging open question is to find alternative, not necessarily open loop, optimal strategies. Then we plan to investigate other optimization techniques, such as model predictive feedbacks and machine learning algorithms. 